\title{
EXPLICIT/IMPLICIT CONSERVATIVE GALERKIN DOMAIN DECOMPOSITION PROCEDURES FOR PARABOLIC PROBLEMS
}

\author{
CLINT N. DAWSON AND TODD F. DUPONT
}

\begin{abstract}
Several domain decomposition methods for approximating solutions of parabolic problems are given. These methods rely on implicit Galerkin procedures in the subdomains and explicit flux calculation on the inter-domain boundaries. The procedures are conservative both in the subdomains and across inter-domain boundaries. A priori error bounds and experimental results are presented.
\end{abstract}

\section{INTRODUCTION}

When solving parabolic partial differential equations using Galerkin finite element procedures, it is frequently advantageous to use implicit time-stepping because of the severe time-step constraint needed to insure stability when using explicit time-stepping. Thus, a large, global system of equations must be solved at each time step. Domain decomposition procedures can be used to break these large computations into several smaller ones, but the smaller "subdomain problems" must be coupled in some way. The procedures studied here use simple, explicit calculations on the boundaries between subdomains to predict the flux, and this is the only coupling between subproblems. Thus, these procedures are noniterative, and involve dividing the domain into nonoverlapping subdomains. The explicit nature of the flux prediction induces a time step limitation that is necessary to preserve stability, but this constraint is less severe than that which comes with a fully explicit method.

Galerkin procedures are useful in situations that require nonrectangular geometry, and the procedures given here allow considerable geometric flexibility. The function spaces used on the subdomains need not match up in such a way that they are restrictions of the same global subspace of $H^{1}$, and the operator used to approximate the flux does not have to match the grids used in the subdomains.

Received October 25, 1990.

1991 Mathematics Subject Classification. Primary 65M60, 65P05.

Key words and phrases. Domain decomposition, parabolic equations, finite elements, parallel computing.

The first author's research was supported by NSF Grant No. DMS-8807257 and used the CRAY2 at the National Center for Supercomputing Applications at the University of Illinois at UrbanaChampaign. 
The a priori error bounds given here rely on previous work on elliptic Galerkin approximations. In fact, our theorems are stated in terms of the errors of certain elliptic approximations rather than powers of some asymptotic parameter.

We exhibit second- and fourth-order correct boundary flux approximations. The analysis is done for arbitrary-order correct boundary flux approximations. The first results use a first-order time discretization, but the results in $\S 4$ give a second-order in time approximation.

Experimental results are also given. They show that the stability constraint is necessary, although not necessarily sharp. They also exhibit (on some example problems) asymptotic rates of convergence that are slightly better than are proved here. These experimental rates of convergence agree with what can be proved in some special cases.

In a previous paper on finite differences [3], we coupled subdomains by explicit predictions of boundary values, and this work was carried over to a finite element context in [2]. The new procedures given here offer greater geometric generality. They also have the advantage that they are conservative across the subdomains boundaries in the sense that the interior boundaries do not serve as sources or sinks. This conservation property is missing in our earlier schemes based on explicit interface value prediction.

\section{Preliminaries}

Let $\Omega$ denote a spatial domain in $\mathbb{R}^{d}$. Denote by $H^{m}(\Omega)$ and $W_{\infty}^{m}(\Omega)$ the standard Sobolev spaces on $\Omega$, with norms $\|\cdot\|_{m}$ and $\|\cdot\|_{\infty, m}$, respectively. Let $L^{p}(\Omega), p=2, \infty$, denote the standard Banach spaces, with $\|\cdot\|$ denoting the $L^{2}$ norm and $\|\cdot\|_{\infty}$ the $L^{\infty}$ norm.

Let $[\alpha, \beta] \subset[0, T]$ denote a time interval and $X=X(\Omega)$ a normed space. To incorporate time-dependence, we use the notation $\|\cdot\|_{L^{p}(\alpha, \beta ; X)}$ to denote the norm of $X$-valued functions $f$ with the map $t \mapsto\|f(\cdot, t)\|_{X}$ belonging to $L^{p}(\alpha, \beta)$.

Assume $\Omega$ has a piecewise uniformly smooth Lipschitz boundary, $\partial \Omega$. Assume that $u^{0}, a$, and $b$ are smooth, real-valued functions on $\bar{\Omega}$, with $a$ being positive and $b$ nonnegative. For some $T>0$, the function $u(x, t)$ satisfies

$$
\begin{aligned}
\frac{\partial u}{\partial t}-\nabla \cdot(a \nabla u)+b u & =0 & & \text { on } \Omega \times(0, T], \\
\frac{\partial u}{\partial n_{\Omega}} & =0 & & \text { on } \partial \Omega \times(0, T], \\
u(x, 0) & =u^{0}(x) & & \text { on } \Omega,
\end{aligned}
$$

where $n_{\Omega}$ is the outward normal to $\partial \Omega$.

We use approximations of derivatives of delta functions at several points in this work, and these approximations can be viewed as coming from onedimensional approximations of the delta function. For future reference we define two special functions $\phi_{2}$ and $\phi_{4}$ as follows:

$$
\phi_{2}(x)= \begin{cases}1-x, & 0 \leq x \leq 1 \\ x+1, & -1 \leq x \leq 0 \\ 0, & \text { otherwise }\end{cases}
$$




$$
\phi_{4}(x)= \begin{cases}(x-2) / 12, & 1 \leq x \leq 2 \\ -5 x / 4+7 / 6, & 0 \leq x \leq 1 \\ 5 x / 4+7 / 6, & -1 \leq x \leq 0 \\ -(x+2) / 12, & -2 \leq x \leq-1 \\ 0, & \text { otherwise }\end{cases}
$$

Note that if $p(x)$ is a polynomial of degree at most one, then

$$
\int p(x) \phi_{2}(x) d x=p(0),
$$

and if $p$ is a polynomial of degree at most three, then

$$
\int p(x) \phi_{4}(x) d x=p(0) \text {. }
$$

\section{DOMAIN DECOMPOSITION PROCEDURES}

In this section we consider the case of two subdomains, and we use a Galerkin procedure on each subdomain. As motivation for the abstract formulation presented later we start with an example. For this example, use $a \equiv b \equiv 1$ in (2.1).

Let $\Omega=(0,1) \times(0,1)$. Take $\Omega_{1}=\left(0, \frac{1}{2}\right) \times(0,1), \Omega_{2}=\left(\frac{1}{2}, 1\right) \times(0,1)$, and $\Gamma=\left\{\frac{1}{2}\right\} \times(0,1)$. Define, for some $H \in\left(0, \frac{1}{2}\right)$,

$$
\phi(x)=\phi_{2}\left(\left(x-\frac{1}{2}\right) / H\right) / H .
$$

For $j=1,2$, let $\mathscr{M}_{j}$ be a finite-dimensional subspace of $H^{1}\left(\Omega_{j}\right)$, and let $\mathscr{M}$ be the subspace of $L^{2}(\Omega)$ such that if $v \in \mathscr{M}$, then $\left.v\right|_{\Omega_{j}} \in \mathscr{M}_{j}$. Note that functions $v$ in $\mathscr{M}$ have a well-defined jump $[v]$ on $\Gamma$; $[v]\left(\frac{1}{2}, y\right)=$ $v\left(\frac{1}{2}+0, y\right)-v\left(\frac{1}{2}-0, y\right)$.

Take the bilinear form $D$ to be given by

$$
D(\psi, \rho)=\sum_{i=1}^{2} \int_{\Omega_{i}}(\nabla \psi \cdot \nabla \rho+\psi \rho) d x d y .
$$

Define an approximate derivative as follows:

$$
B(\psi)\left(\frac{1}{2}, y\right)=-\int_{0}^{1} \phi^{\prime}(x) \psi(x, y) d x,
$$

where $\phi$ is given by (3.1).

Let $(\cdot, \cdot)_{\Sigma}$ be the $L^{2}(\Sigma)$ inner product,

$$
(\psi, \rho)_{\Sigma}=\int_{\Sigma} \psi \rho .
$$

In the case $\Sigma=\Omega$ we omit the subscript:

$$
(\psi, \rho)=(\psi, \rho)_{\Omega} .
$$

Let $0=t^{0}<t^{1}<\cdots<t^{M}=T$ be a given sequence, and suppose that $U^{0} \in \mathscr{M}$ is given. Define $U^{1}, \ldots, U^{M}$ by

$$
\left(\partial_{t} U^{n}, v\right)+D\left(U^{n}, v\right)+\left(B\left(U^{n-1}\right),[v]\right)_{\Gamma}=0, \quad v \in \mathscr{M},
$$


where $\Delta t^{n}=t^{n}-t^{n-1}$ and

$$
\partial_{t} U^{n}=\left(U^{n}-U^{n-1}\right) / \Delta t^{n}
$$

This scheme has the property that $U^{n}$ can be computed on $\Omega_{1}$ and $\Omega_{2}$ completely independently once $B\left(U^{n-1}\right)$ has been computed on $\Gamma$. The flux at each point on $\Gamma$ is computed explicitly from $U^{n-1}$, and the two parts of $U^{n}$ are then computed using an implicit Galerkin backward difference equation on each $\Omega_{j}$. Note also that if the function $v \equiv 1$ belongs to $\mathscr{M}_{j}$, then (3.6) is conservative in the following sense. If we use $b \equiv 0$ instead of 1 , then the average value of $U^{n}$ is the same for all $n$, just as the average value of $u(\cdot, t)$ is independent of $t$, by (2.1) and (2.2).

For functions $\psi$ with restrictions in $H^{1}\left(\Omega_{1}\right)$ and $H^{1}\left(\Omega_{2}\right)$, define

$$
\|\mid \psi\|\left\|^{2}=D(\psi, \psi)+H^{-1}\right\|[\psi] \|_{L^{2}(\Gamma)}^{2} .
$$

Next, note that for such $\psi$ 's,

$$
\begin{aligned}
(B(\psi),[\psi])_{\Gamma}= & -\int_{0}^{1} \int_{0}^{1} \phi^{\prime}(x) \psi(x, y)[\psi]\left(\frac{1}{2}, y\right) d x d y \\
= & H^{-1} \int_{0}^{1}[\psi]^{2}\left(\frac{1}{2}, y\right) d y \\
& +\int_{0}^{1} \int_{0}^{1} \phi(x) \psi_{x}(x, y) d x[\psi]\left(\frac{1}{2}, y\right) d y
\end{aligned}
$$

and

$$
\begin{aligned}
& \int_{0}^{1} \int_{0}^{1} \phi(x) \psi_{x}(x, y) d x[\psi]\left(\frac{1}{2}, y\right) d y \\
& \quad \leq\left\|\psi_{x}\right\|_{L^{2}\left(\Omega_{1} \cup \Omega_{2}\right)}\|\phi\|_{L^{2}(0,1)}\|[\psi]\|_{L^{2}(\Gamma)} \\
& \quad \leq\left\|\psi_{x}\right\|_{L^{2}\left(\Omega_{1} \cup \Omega_{2}\right)}\left((2 / 3 H)^{1 / 2}\|[\psi]\|_{L^{2}(\Gamma)}\right) .
\end{aligned}
$$

From this result we get that

$$
D(\psi, \psi)+(B(\psi),[\psi])_{\Gamma} \geq(1-1 / \sqrt{6}) \mid\|\psi\| \|^{2} ;
$$

in deriving this, we used

$$
\alpha \beta \leq \varepsilon \alpha^{2}+\beta^{2} / 4 \varepsilon
$$

on the product in (3.10) with $\varepsilon^{2}=\frac{1}{6}$.

We restate $(3.11)$ as

$$
\begin{aligned}
\|\psi \psi\|^{2} & \leq(1-1 / \sqrt{6})^{-1}\left(D(\psi, \psi)+(B(\psi),[\psi])_{\Gamma}\right) \\
& <1.7\left(D(\psi, \psi)+(B(\psi),[\psi])_{\Gamma}\right) .
\end{aligned}
$$

The following two bounds are straightforward:

$$
\begin{aligned}
\|B(\psi)\|_{L^{2}(\Gamma)}^{2} & \leq 2 H^{-3}\|\psi\|^{2}, \\
\|B(\psi)\|_{L^{2}(\Gamma)} & \leq 2 H^{-1}\|\psi\|_{\infty} .
\end{aligned}
$$

Also, if $\psi$ is smooth in $\Omega$, it follows from integration by parts, $(2.4)$, and Taylor's Theorem that

$$
B(\psi)\left(\frac{1}{2}, y\right)=\psi_{x}\left(\frac{1}{2}, y\right)+\int_{0}^{1} \int_{1 / 2}^{x}(x-s) \psi_{x x x}\left(\frac{1}{2}, s\right) d s \phi(x) d x .
$$


Hence, it follows

$$
\left\|\psi_{x}\left(\frac{1}{2}, \cdot\right)-B(\psi)\right\|_{L^{2}(\Gamma)} \leq \frac{1}{12} H^{2}\left\|\psi_{x x x}\right\|_{\infty} .
$$

To state an error bound for the procedure (3.6), we introduce an elliptic projection [6] $W \in \mathscr{M}$ of the solution $u$ as follows:

$$
D(u(\cdot, t)-W(\cdot, t), v)=0, \quad v \in \mathscr{M} .
$$

The function $W$ at each $t$ is just the $H^{1}\left(\Omega_{j}\right)$ projection of $u$ into $\mathscr{M}_{j}$. Let the error in the projection be denoted by

$$
\eta=u-W
$$

Theorem 1. Suppose that the solution $u$ is sufficiently smooth and that $U^{0} \in \mathscr{M}$ is taken to be $W^{0}$. Let $\Delta t=\max _{n} \Delta t^{n}$. Then there exists a constant $C$, independent of the spaces $\mathscr{M}_{j}$, such that

$$
\begin{aligned}
\max _{n} & \left\|u^{n}-U^{n}\right\| \\
& \leq C\left(\Delta t+H^{2.5}+\int_{0}^{T}\left\|\eta_{t}(\cdot, t)\right\| d t+H^{-1 / 2}\|\eta\|_{L^{\infty}(\Omega \times(0, T))}\right),
\end{aligned}
$$

provided that

$$
\Delta t \leq H^{2} / 4 \text {. }
$$

The procedure is first-order correct in $\Delta t$ as expected, since this would be the case even if no domain decomposition were used. The second-order correct approximation $B$ gives rise to the $H^{2.5}$, since we only make the $H^{2}$ error on a "small" set. The loss of $H^{-1 / 2}$ in the last term in the estimate can be avoided in certain special cases using the techniques of [1], but at this level of generality we do not know how to improve this term. The theorem will follow as an easy consequence of Theorem 2 below.

We now treat a more general case. The domain $\Omega$ in $\mathbb{R}^{d}$ is divided into two nonoverlapping subdomains $\Omega_{1}$ and $\Omega_{2}$. The interface between these domains

$$
\Gamma=\Omega \cap \bar{\Omega}_{1} \cap \bar{\Omega}_{2}
$$

is assumed to be a uniformly smooth $(d-1)$-dimensional manifold.

For functions $\psi, \rho$ with restrictions in $H^{1}\left(\Omega_{j}\right)$, let

$$
D(\psi, \rho)=\int_{\Omega_{1} \cup \Omega_{2}}(a \nabla \psi \cdot \nabla \rho+b \psi \rho) d x,
$$

and let the jump in such a $\psi$ on $\Gamma$ be denoted by $[\psi]$. For definiteness, let $[\psi]$ be the trace from $\Omega_{2}$ minus the trace from $\Omega_{1}$.

We need a parametrized approximation to the normal derivative on $\Gamma$. Assume for some $H>0$ that $B$ is a linear map of $L^{2}(\Omega)$ into $L^{2}(\Gamma)$ and that it satisfies the following four conditions, which are generalizations of (3.13), (3.14), (3.15), and (3.17):

(i) There is a constant $C_{0}$ such that

$$
\|\mid \psi\| \|^{2} \leq C_{0}\left(D(\psi, \psi)+(a B(\psi),[\psi])_{\Gamma}\right)
$$

where

$$
\|\psi \psi\| \|^{2}=D(\psi, \psi)+H^{-1}(a[\psi],[\psi])_{\Gamma} .
$$


(ii) There is a constant $C_{1}$ such that

$$
(a B(\psi), B(\psi))_{\Gamma} \leq C_{1} H^{-3}\|\psi\|^{2} .
$$

(iii) There is a constant $C_{2}$ such that

$$
\|B(\psi)\|_{L^{2}(\Gamma)} \leq C_{2} H^{-1}\|\psi\|_{L^{\infty}(\Omega)} .
$$

(iv) There is a constant $k \geq 0$ and a constant $C_{3}$ which depends on the solution $u$ such that

$$
\left\|\frac{\partial u(\cdot, t)}{\partial \gamma}-B(u)(\cdot, t)\right\|_{L^{2}(\Gamma)} \leq C_{3} H^{k}
$$

for $0 \leq t \leq T$, where $\partial u / \partial \gamma$ is the normal derivative of $u$ on $\Gamma$, in the direction from $\Omega_{1}$ to $\Omega_{2}$.

Suppose that $\mathscr{M}_{j}$ is a finite-dimensional subspace of $H^{1}\left(\Omega_{j}\right)$, and let be the set of $L^{2}(\Omega)$ functions whose restrictions to $\Omega_{j}$ belong to $\mathscr{M}_{j}$. For $0=t^{0}<t^{1}<\cdots<t^{M}=T$ and $U^{0} \in \mathscr{M}$ given, define $U^{1}, \ldots, U^{M}$ by

$$
\left(\partial_{t} U^{n}, v\right)+D\left(U^{n}, v\right)+\left(a B\left(U^{n-1}\right),[v]\right)_{\Gamma}=0, \quad v \in \mathscr{M},
$$

where $\partial_{t} U^{n}$ is given by (3.7).

Just as in the example, we define $W(\cdot, t) \in \mathscr{M}$ by

$$
D((u-W)(\cdot, t), v)=0, \quad v \in \mathscr{M},
$$

at each $t \in[0, T]$. Let the error in the elliptic projection be denoted by

$$
\eta=u-W \text {. }
$$

Theorem 2. Suppose that the solution $u$ to $(2.1)$ is sufficiently smooth and that $U^{0}=W(\cdot, 0)$. Let $\Delta t=\max _{n} \Delta t^{n}$. Then there exists a constant $C$, independent of the spaces $\mathscr{M}_{j}$, such that

$$
\begin{aligned}
\max _{n} & \left\|(u-U)\left(\cdot, t^{n}\right)\right\| \\
& \leq C\left\{\Delta t+H^{k+1 / 2}+\int_{0}^{T}\left\|\eta_{t}(\cdot, t)\right\| d t+H^{-1 / 2}\|\eta\|_{L^{\infty}(\Omega \times(0, T))}\right\},
\end{aligned}
$$

provided

$$
\Delta t \leq H^{2} / C_{0} C_{1} .
$$

Note that there are no assumptions that require $\mathscr{M}_{1}$ and $\mathscr{M}_{2}$ to be compatible in some way. Also, as we saw in the example, the $H$-parameter for the operator $B$ is not necessarily related to any aspect of the spaces $\mathscr{M}_{j}$; in particular, it is not required that $\phi$ restricted to $\Omega_{j}$ have any relation to $\mathscr{K}_{j}$.

Proof of Theorem 2. Note first that $u$ satisfies

$$
\left(\partial_{t} u^{n}, v\right)+D\left(u^{n}, v\right)+\left(a \frac{\partial u^{n}}{\partial \gamma},[v]\right)_{\Gamma}=\left(\rho^{n}, v\right), \quad v \in \mathscr{M},
$$

where $u^{n}(x)=u\left(x, t^{n}\right)$. The time truncation term $\rho^{n}$ satisfies

$$
\sum_{n=1}^{M}\left\|\rho^{n}\right\| \Delta t^{n} \leq \Delta t \int_{0}^{T}\left\|u_{t t}(\cdot, t)\right\| d t \leq C \Delta t .
$$


From (3.33) and the definition of $W$ we see that

$$
\begin{aligned}
& \left(\partial_{t} W^{n}, v\right)+D\left(W^{n}, v\right)+\left(a B\left(W^{n-1}\right),[v]\right)_{\Gamma} \\
& \quad=\left(\rho^{n}-\partial_{t} \eta^{n}, v\right)+\left(a\left(B\left(W^{n-1}\right)-\frac{\partial u^{n}}{\partial \gamma}\right),[v]\right)_{\Gamma}, \quad v \in \mathscr{M} .
\end{aligned}
$$

Let

$$
\nu=U-W .
$$

This gives, from (3.29) and (3.35),

$$
\begin{aligned}
& \left(\partial_{t} \nu^{n}, v\right)+D\left(\nu^{n}, v\right)+\left(a B\left(\nu^{n-1}\right),[v]\right)_{\Gamma} \\
& \quad=\left(\partial_{t} \eta^{n}-\rho^{n}, v\right)+\left(a\left(\frac{\partial u^{n}}{\partial \gamma}-B\left(W^{n-1}\right)\right),[v]\right)_{\Gamma}, \quad v \in \mathscr{M} .
\end{aligned}
$$

Let

$$
\vartheta=a\left(\frac{\partial u^{n}}{\partial \gamma}-B\left(W^{n-1}\right)\right)
$$

Then

$$
\vartheta=a\left(\frac{\partial u^{n}}{\partial \gamma}-\frac{\partial u^{n-1}}{\partial \gamma}+\frac{\partial u^{n-1}}{\partial \gamma}-B\left(u^{n-1}\right)+B\left(\eta^{n-1}\right)\right) .
$$

From this it follows that

$$
\|\vartheta\|_{L^{2}(\Gamma)} \leq C\left(\Delta t+H^{k}+H^{-1}\left\|\eta^{n-1}\right\|_{\infty}\right) .
$$

Use $v=\nu^{n}$ in (3.37) to see that

$$
\begin{aligned}
\left(\partial_{t} \nu^{n},\right. & \left.\nu^{n}\right)+D\left(\nu^{n}, \nu^{n}\right)+\left(a B\left(\nu^{n}\right),\left[\nu^{n}\right]\right)_{\Gamma} \\
\leq & \left(a B\left(\nu^{n}-\nu^{n-1}\right),\left[\nu^{n}\right]\right)_{\Gamma}+\left\|\partial_{t} \eta^{n}+\rho^{n}\right\|\left\|\nu^{n}\right\| \\
& +C\left(\Delta t+H^{k}+H^{-1}\left\|\eta^{n-1}\right\|_{\infty}\right) H^{1 / 2}\left\|\nu^{n}\right\| .
\end{aligned}
$$

Since $(\alpha-\beta) \alpha=\frac{1}{2}\left(\alpha^{2}-\beta^{2}\right)+\frac{1}{2}(\alpha-\beta)^{2}$, we see that

$$
\left(\partial_{t} \nu^{n}, \nu^{n}\right)=\frac{1}{2} \partial_{t}\left(\left\|\nu^{n}\right\|^{2}\right)+\frac{\Delta t^{n}}{2}\left\|\partial_{t} \nu^{n}\right\|^{2} .
$$

Use this and (3.24) in (3.40) to see that

$$
\begin{aligned}
& \partial_{t}\left(\left\|\nu^{n}\right\|^{2}\right)+\Delta t^{n}\left\|\partial_{t} \nu^{n}\right\|^{2}+\left.\frac{2}{C_{0}}\left\|\nu^{n}\right\|\right|^{2} \\
& \leq 2 C_{1}^{1 / 2} H^{-1} \Delta t^{n}\left\|\partial_{t} \nu^{n}\right\|\left\|\nu^{n}\right\|+2\left\|\partial_{t} \eta^{n}+\rho^{n}\right\|\left\|\nu^{n}\right\| \\
&+C\left(\Delta t+H^{k}+H^{-1}\left\|\eta^{n-1}\right\|_{\infty}\right) H^{1 / 2}\left\|\nu^{n}\right\| \\
& \leq \frac{2}{C_{0}}\left\|\nu^{n}\right\|\left\|^{2}+\left(C_{0} C_{1} H^{-2} \Delta t^{n}\right) \Delta t^{n}\right\| \partial_{t} \nu^{n} \|^{2} \\
&+2\left\|\partial_{t} \eta^{n}+\rho^{n}\right\|\left\|\nu^{n}\right\|+C\left(\Delta t+H^{k}+H^{-1}\left\|\eta^{n-1}\right\|_{\infty}\right)^{2} H .
\end{aligned}
$$

Provided

$$
\Delta t^{n} \leq H^{2} / C_{0} C_{1},
$$

we see that

$$
\begin{aligned}
\partial_{t}\left(\left\|\nu^{n}\right\|^{2}\right) \leq & 2\left\|\partial_{t} \eta^{n}+\rho^{n}\right\|\left\|\nu^{n}\right\| \\
& +C\left(\Delta t^{2} H+H^{2 k+1}+H^{-1}\left\|\eta^{n-1}\right\|_{\infty}^{2}\right) .
\end{aligned}
$$


From this we see that, by multiplying by $\Delta t^{n}$ and summing on $n$,

$$
\begin{aligned}
\left(\max _{n}\left\|\nu^{n}\right\|\right)^{2} \leq & \left\|\nu^{0}\right\|^{2}+2 \sum_{n=1}^{M}\left\|\partial_{t} \eta^{n}+\rho^{n}\right\|\left\|\nu^{n}\right\| \Delta t^{n} \\
& +C\left(\Delta t^{2} H+H^{2 k+1}+H^{-1}\|\eta\|_{L^{\infty}(\Omega \times(0, T))}^{2}\right) .
\end{aligned}
$$

Use $\nu^{0}=0$ and

$$
\begin{gathered}
2 \sum_{n=1}^{M}\left\|\partial_{t} \eta^{n}+\rho^{n}\right\|\left\|\nu^{n}\right\| \Delta t^{n} \leq 2 \max _{n}\left\|\nu^{n}\right\| \sum_{n=1}^{M}\left\|\partial_{t} \eta^{n}+\rho^{n}\right\| \Delta t^{n} \\
\leq \frac{1}{2}\left(\max _{n}\left\|\nu^{n}\right\|\right)^{2}+4\left(\sum_{n=1}^{M}\left\|\partial_{t} \eta^{n}+\rho^{n}\right\| \Delta t^{n}\right)^{2}
\end{gathered}
$$

to see that

$$
\begin{aligned}
\max _{n}\left\|\nu^{n}\right\| \leq C\left(\Delta t+H^{k+1 / 2}+H^{-1 / 2}\|\eta\|_{L^{\infty}(\Omega \times(0, T))}\right. & \\
& \left.+\int_{0}^{T}\left\|\eta_{t}(\cdot, t)\right\| d t\right) .
\end{aligned}
$$

Here we have also used (3.34). The conclusion follows from the triangle inequality.

The bound (3.43) (and hence (3.32)) can be relaxed by almost a factor of two, at the expense of a larger constant in the last term (3.42). The $L^{\infty}$ bound on $\eta$ is needed near $\Gamma$ in the examples of $B$ with which we have worked; away from $\Gamma$, an $L^{2}$ bound can be used instead.

Note that the projection $W$ has no dependence on $H$, and this implies that $\eta$ has no $H$-dependence. From this observation it follows that we can allow $H$ to vary from step to step, provided only that

$$
\Delta t^{n} \leq H_{n}^{2} / C_{0} C_{1} .
$$

Return to consideration of the example problem used to introduce this section. Define $B$ using $\phi_{4}$ of $\S 2$ by taking $\phi(x)=\phi_{4}\left(\left(x-\frac{1}{2}\right) / H\right) / H$. Now we need $H \in\left(0, \frac{1}{4}\right)$. Then calculation gives that (3.24), (3.26), and (3.27) hold with $C_{0}=1.64, C_{1}=3.14$, and $C_{2}=\frac{8}{3}$. Thus, Theorem 2 requires $\Delta t \leq H^{2} / 5.15$. In this case (3.28) holds with $k=4$.

\section{A SECOND-ORDER IN TIME PROCEDURE}

In this section we illustrate the use of a second-order in time backward discretization using the Galerkin-based procedure of $\S 3$. We restrict attention to the case of uniform time steps $t^{n}=n \Delta t$. The context used here is that of Theorem 2; i.e., we allow variable coefficients, $\Omega \subset \mathbb{R}^{d}$, and $\Gamma$ is a smooth $(d-1)$-dimensional manifold.

The results of this section are slightly related to the results in [5], where energy methods are used on second-order backward difference Galerkin methods (and blending methods). In [5], variable time steps are analyzed, but not in a domain decomposition context. 
Let

$$
\delta_{2} U^{n}=\partial_{t} U^{n}+\frac{1}{2}\left(\partial_{t} U^{n}-\partial_{t} U^{n-1}\right) .
$$

Assume that $U^{0}$ and $U^{1}$ in $\mathscr{M}$ are known, and define $U^{2}, \ldots, U^{M}$ by the following analogue of (3.29):

$$
\left(\delta_{2} U^{n}, v\right)+D\left(U^{n}, v\right)+\left(B\left(2 U^{n-1}-U^{n-2}\right),[v]\right)_{\Gamma}=0, \quad v \in \mathscr{M} .
$$

This procedure is formally second-order correct in time, and like (3.29), it allows the calculation of $U^{n}$ to be done independently on $\Omega_{1}$ and $\Omega_{2}$ once the operator $B$ has been evaluated on $\Gamma$ using $2 U^{n-1}-U^{n-2}$.

The analogue of Theorem 2 is the following:

Theorem 3. Take $W$ and $\eta$ as in (3.30) and (3.31) and let $B$ satisfy (3.24), (3.26), (3.27), and (3.28). Suppose that the solution $u$ of (2.1) is sufficiently smooth and that $U^{0}=W(\cdot, 0)$ and $U^{1}=W(\cdot, \Delta t)$. Then there is a constant $C$, independent of the spaces $\mathscr{M}_{j}$, such that $U^{n}$ given by (4.2) satisfies

$$
\begin{aligned}
\max _{n} & \left\|(u-U)\left(\cdot, t^{n}\right)\right\| \\
& \leq C\left\{\Delta t^{2}+H^{k+1 / 2}+\int_{0}^{T}\left\|\eta_{t}(\cdot, t)\right\| d t+H^{-1 / 2}\|\eta\|_{L^{\infty}(\Omega \times(0, T))}\right\},
\end{aligned}
$$

provided

$$
\Delta t \leq H^{2} / 2 C_{0} C_{1} \text {. }
$$

Of course, it is unlikely that we could take $U^{0}$ and $U^{1}$ exactly as indicated in the theorem. The proof will show that $U^{0}$ and $U^{1}$ need only approximate these values in a certain sense, but we will not give the details of that result.

Proof of Theorem 3. In analogy with (3.33) we see that

$$
\left(\delta_{2} u^{n}, v\right)+D\left(u^{n}, v\right)+\left(a \frac{\partial u^{n}}{\partial \gamma},[v]\right)_{\Gamma}=\left(\rho^{n}, v\right), \quad v \in \mathscr{M}
$$

where

$$
\sum_{n=1}^{M}\left\|\rho^{n}\right\| \Delta t \leq C(\Delta t)^{2} \int_{0}^{T}\left\|u_{t t t}(\cdot, t)\right\| d t \leq C(\Delta t)^{2}
$$

Thus,

$$
\begin{aligned}
& \left(\delta_{2} W^{n}, v\right)+D\left(W^{n}, v\right)+\left(a B\left(2 W^{n-1}-W^{n-2}\right),[v]\right)_{\Gamma} \\
& =\left(\rho^{n}-\delta_{2} \eta^{n}, v\right)+\left(a\left(B\left(2 W^{n-1}-W^{n-2}\right)-\frac{\partial u^{n}}{\partial \gamma}\right),[v]\right)_{\Gamma}, \\
& v \in \mathscr{M}_{j} .
\end{aligned}
$$

Use

$$
\begin{aligned}
\frac{\partial u^{n}}{\partial \gamma}- & B\left(2 W^{n-1}-W^{n-2}\right) \\
= & \frac{\partial\left(u^{n}-2 u^{n-1}+u^{n-2}\right)}{\partial \gamma}+\left[\frac{\partial\left(2 u^{n-1}-u^{n-2}\right)}{\partial \gamma}-B\left(2 u^{n-1}-u^{n-2}\right)\right] \\
& +B\left(2 \eta^{n-1}-\eta^{n-2}\right)
\end{aligned}
$$


to get

$$
\begin{aligned}
\left(\delta_{2} \nu^{n},\right. & \left.\nu^{n}\right)+D\left(\nu^{n}, \nu^{n}\right)+\left(a B\left(\nu^{n}\right),\left[\nu^{n}\right]\right)_{\Gamma} \\
= & \left(a B\left(\nu^{n}-2 \nu^{n-1}+\nu^{n-2}\right),\left[\nu^{n}\right]\right)_{\Gamma}+\left\|\delta_{2} \eta^{n}+\rho^{n}\right\|\left\|\nu^{n}\right\| \\
& +C\left((\Delta t)^{2}+H^{k}+H^{-1}\left(\left\|\eta^{n-1}\right\|_{\infty}+\left\|\eta^{n-2}\right\|_{\infty}\right)\right) H^{1 / 2}\left\|\mid \nu^{n}\right\| \| .
\end{aligned}
$$

To replace $(3.41)$, we use

$$
\left(\delta_{2} \nu^{n}, \nu^{n}\right)=\partial_{t} E\left(\nu^{n}, \nu^{n-1}\right)+\frac{1}{4 \Delta t}\left(\nu^{n}-2 \nu^{n-1}+\nu^{n-2}\right)^{2},
$$

where

$$
E(\psi, \mu)=\frac{1}{4}\left(\|\psi\|^{2}+\|2 \psi-\mu\|^{2}\right) \geq \frac{1}{4}\|\psi\|^{2} .
$$

The first term on the right side of $(4.8)$ is bounded by

$$
\begin{aligned}
& \frac{C_{0} H}{2}\left(a B\left(\nu^{n}-2 \nu^{n-1}+\nu^{n-2}\right), B\left(\nu^{n}-2 \nu^{n-1}+\nu^{n-2}\right)\right)_{\Gamma}+\frac{1}{2 C_{0}}\left\|\nu^{n}\right\| \|^{2} \\
& \leq \frac{C_{0} H}{2} C_{1} H^{-3}\left\|\nu^{n}-2 \nu^{n-1}+\nu^{n-2}\right\|^{2}+\frac{1}{2 C_{0}}\left\|\nu^{n}\right\| \|^{2},
\end{aligned}
$$

where (3.26) was used to introduce $C_{1}$. To complete the proof, follow the proof of Theorem 2.

The critical step in determining the $\Delta t$-constraint is the relation

$$
\frac{1}{4 \Delta t}-\left(\frac{C_{0} H}{2}\right)\left(\frac{C_{1}}{H^{3}}\right) \geq 0 \text {. }
$$

This gives

$$
\Delta t \leq H^{2} / 2 C_{0} C_{1} ;
$$

just as in Theorem 2, this could be relaxed by almost a factor of two.

\section{NUMERICAL EXPERIMENTS}

In this section we present the results of some numerical experiments for the Galerkin procedure described above.

First, we study the sensitivity of the scheme to the $\Delta t-H$ constraint. Consider (2.1) on the unit interval in $\mathbb{R}$ with $a=1$ and $b=0$. Recall that, for purposes of $L^{2}$-stability, the constraint is of the form

$$
\Delta t<H^{2} / 2 \text {. }
$$

We take as initial data the function $u^{0}$ given in Figure 1. At steady-state, the solution $u(x, t) \equiv \int_{0}^{1} u^{0}(x)$. We apply the Galerkin procedure (3.3), (3.6) to this problem, taking four subdomains. The $L^{2}$ norm of the solution for two different values of $\Delta t / H^{2}$ is given in Figure 2 . Here it can be seen that when (5.1) is violated by as much as a factor of two, $\|U(\cdot, t)\|$ blows up as time increases.

Next, we study the experimental rate of convergence of the scheme. As remarked earlier, in certain instances a better rate of convergence than that predicted by Theorem 2 can be proven. However, for piecewise linear approximating spaces and $B(\psi)$ based on $\phi_{k}, k=2$ or 4 , we expect the rate of convergence to be at best quadratic in $h$ and order $k+1$ in $H$, based on the truncation 


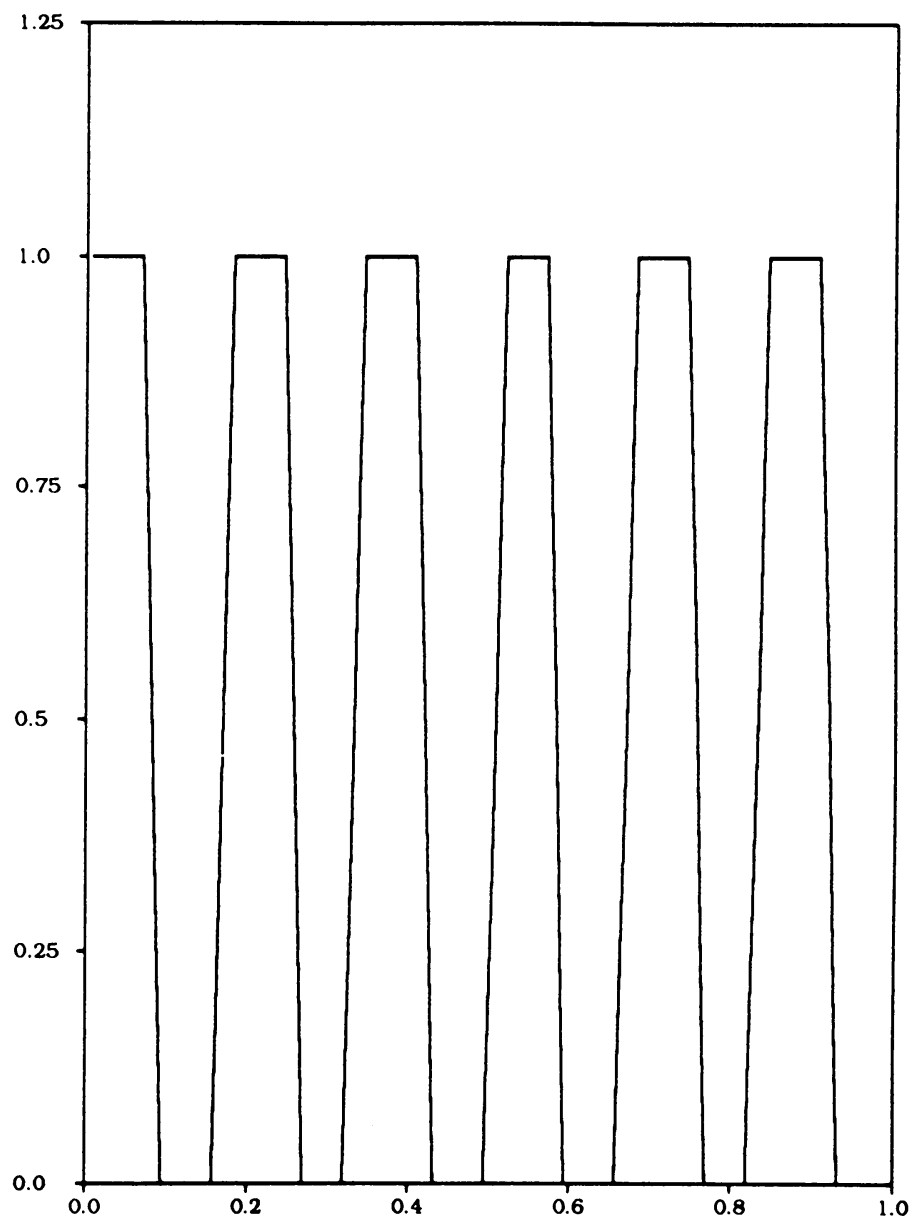

FIGURE 1

Initial data for stability test

error of the scheme and the fact that the support of $\phi_{k}$ is $\mathscr{O}(H)$. Determining the sharpness of the estimates in general will be the subject of future work.

In these runs, $a=1$ and $b=0$, and $\Omega=(0,1) \times(0,1)$. The solution $u$ is approximated in the space of continuous piecewise linears in $x$ tensored with continuous piecewise linears in $y$. We consider three scenarios:

1. Fully implicit Galerkin on uniform mesh; i.e., no domain decomposition;

2. Galerkin domain decomposition with four subdomains, with interfaces $\Gamma^{i}=\{i / 4\} \times(0,1), i=1,2,3$, and with global uniform mesh;

3. Galerkin domain decomposition with three subdomains $\Omega_{i}, i=1,2,3$, with interfaces $\Gamma^{1}=\{0.3\} \times(0,1)$ and $\Gamma^{2}=\{0.55\} \times(0,1)$. The mesh is uniform in $y$ and in $x$ on each subdomain but not uniform across subdomains.

First, consider

$$
\begin{gathered}
u_{t}-\Delta u=0, \\
u^{0}(x, y)=u_{1}^{0}(x, y)=\cos (2 \pi x) \cos (\pi y),
\end{gathered}
$$




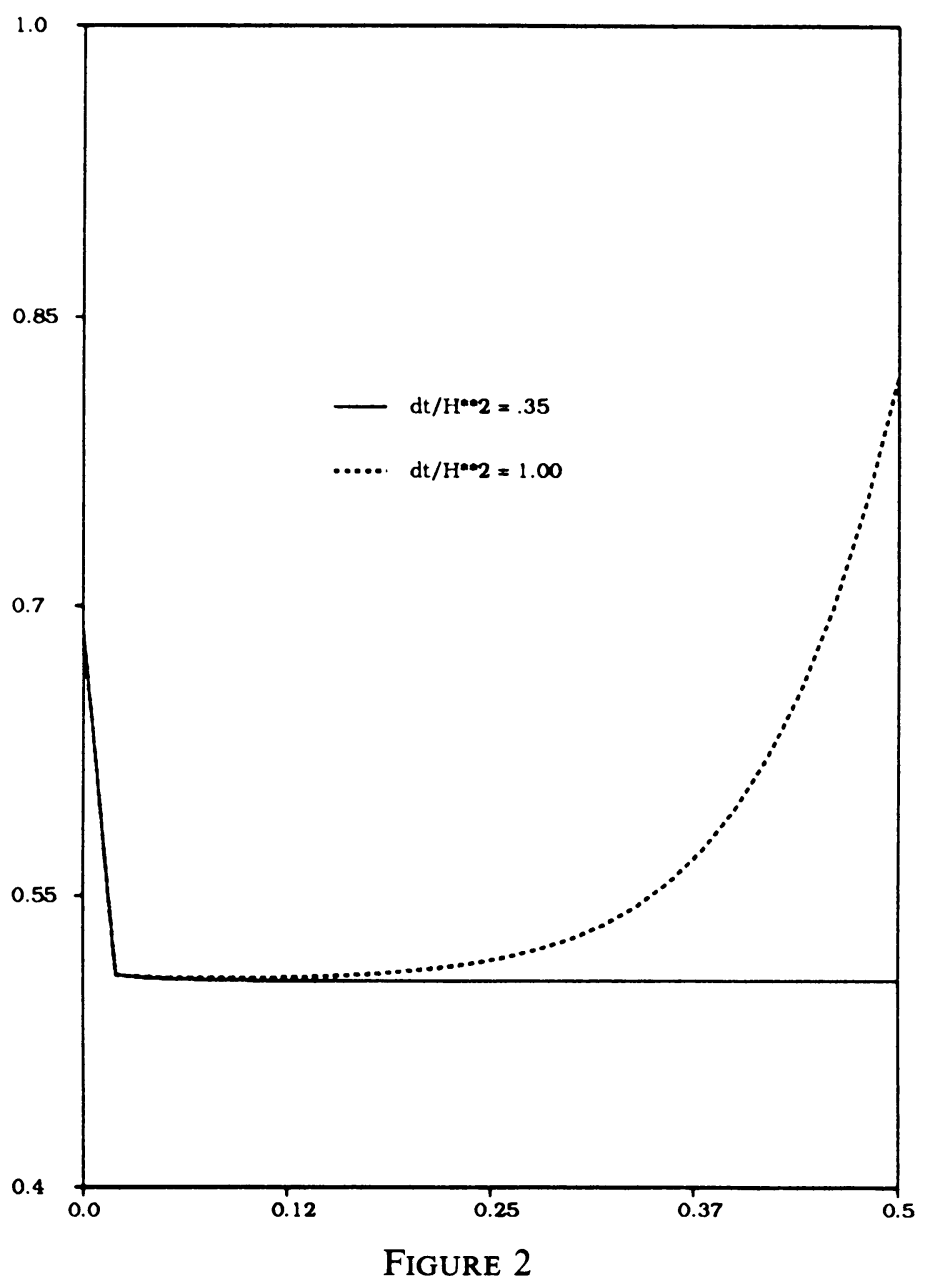

Stability of domain decomposition solution: $\|U\|$ vs. time

which gives the solution $u(x, y, t) \equiv u_{1}(x, y, t)=e^{-5 \pi^{2} t} u_{1}^{0}(x, y)$. We approximate $u$ on $40 \times 40,80 \times 80$, and $160 \times 160$ grids. In the third scenario above, the coarsest mesh in the $x$-direction is $\frac{3}{160}$ in $\Omega_{1}, \frac{1}{32}$ in $\Omega_{2}$, and $\frac{9}{320}$ in $\Omega_{3}$. All subsequent meshes are obtained by halving this mesh. In Table 1 , we give the $L^{2}$ error for $e_{h}=u-U$ for each scenario listed above, at time $T=.03$. In these runs, $\Delta t=4 h^{2}$, and $H=4 h$. In each case, the error is approaching second order as the grid is refined; however, as seen in Table $1, h$ needs to be smaller to get into the asymptotic range for the domain decomposition approach. The rate here is computed by observing that $e_{h} \approx C h^{q}$ and doing a least squares fit to determine $q$. It is curious to note that the errors in the domain decomposition cases are smaller than the errors for the fully implicit scheme.

In Table 2, we compare the error obtained when computing the derivative using $\phi_{4}$ versus $\phi_{2}$, for the three-subdomain case. Here we see that, in this particular case at least, the overall error is not reduced substantially by the extra accuracy of $\phi_{4}$. 
TABLE 1

Convergence in $h: u(x, t)=u_{1}(x, y, t)$

Implicit

DD-4 s.d.

DD-3 s.d.

\begin{tabular}{||r|c|c|c|c|c|c||}
\hline$h^{-1}, H$ & $e_{h} * 10^{4}$ & Rate & $e_{h} * 10^{4}$ & Rate & $e_{h} * 10^{4}$ & Rate \\
\hline $40, .1$ & 94.24 & - & 54.50 & - & 74.95 & - \\
\hline $80, .05$ & 24.30 & - & 18.60 & - & 21.37 & - \\
\hline $160, .025$ & 6.11 & 1.97 & 5.38 & 1.67 & 5.71 & 1.88 \\
\hline $320, .0125$ & - & - & 1.44 & 1.75 & - & - \\
\hline
\end{tabular}

TABLE 2

$\phi_{2}$ vs. $\phi_{4}: u(x, y, t)=u_{1}(x, y, t)$

DD-3 s.d.

\begin{tabular}{||r|c|c||}
\hline$h^{-1}$ & $e_{h} * 10^{4}: \phi_{2}$ & $e_{h} * 10^{4}: \phi_{\mathbf{4}}$ \\
\hline 40 & 77.60 & 70.10 \\
\hline 80 & 21.60 & 19.70 \\
\hline 160 & 5.71 & 5.70 \\
\hline
\end{tabular}

TABLE 3

Convergence in $h: u(x, y, t)=u_{2}(x, y, t)$

Implicit

DD-4 s.d.

DD-3 s.d.

\begin{tabular}{||r|c|c|c|c|c|c||}
\hline$h^{-1}, H$ & $e_{h} * 10^{4}$ & Rate & $e_{h} * 10^{4}$ & Rate & $e_{h} * 10^{4}$ & Rate \\
\hline $20, .1$ & 80.65 & - & 79.90 & - & 85.07 & - \\
\hline $40, .05$ & 20.20 & - & 19.20 & - & 21.25 & - \\
\hline $80, .025$ & 5.05 & 2.00 & 4.87 & 2.00 & 5.37 & 2.00 \\
\hline
\end{tabular}

Next, consider

$$
u_{t}-\Delta u=f(x, y, t), \quad u^{0}(x, y)=0,
$$

where $f$ is chosen so that $u(x, y, t) \equiv u_{2}(x, y, t)=100 t x^{3}(1-x)^{2} \cos (2 \pi y)$. We perform similar experiments in this case, with final time $T=.5$. The results are given in Table 3. Again, second-order convergence in $h$ is seen.

We now study the rate of convergence in $H$. Return to (5.2) and consider the third scenario above, with three subdomains. We take $h^{-1}=320$ and $\Delta t=4 / 320^{2}$. The underlying mesh in $\Omega_{1}$ and $\Omega_{3}$ is $128 \times 320$, while the underlying mesh in $\Omega_{2}$ is $64 \times 320$. We compare the errors for $H^{-1}=5,10$, and 20 in Table 4. Assume the error is of the form

$$
e_{H}=e_{h}+C H^{p} \text {. }
$$




\section{TABLE 4}

Convergence in $H: u(x, y, t)=u_{1}(x, y, t)$

DD-3 s.d.

\begin{tabular}{||r|c||}
\hline$H^{-1}$ & $e_{H} * 10^{4}$ \\
\hline 5 & 94.40 \\
\hline 10 & 12.80 \\
\hline 20 & 2.52 \\
\hline
\end{tabular}

By numerical experimentation, letting $H \rightarrow 0$, we found $e_{h} \approx 1.48 * 10^{-4}$. Performing a least squares fit of the error based on (5.4),

$$
e_{H} \approx 1.48 * 10^{-4}+1.80 H^{3.24} \text {. }
$$

Thus, cubic accuracy in $H$ is observed.

In conclusion, for the test problems presented here, the method exhibits better accuracy than predicted by the theorems. In the case of uniform global mesh, such as used in scenario 2 , the improvement in $h$ can be explained by arguments given in [1], as mentioned earlier. The improvement in $H$ can be explained by one-dimensional arguments given in [4]. The improved convergence rates for the third scenario are more mysterious, however. In future work, we will attempt to address these and other issues. The point to note is that the algorithm performs quite well and is a viable procedure for solving parabolic equations on coarse-grain parallel computers.

\section{BIBLIOGRAPHY}

1. J. H. Bramble and A. H. Schatz, Higher order local accuracy by averaging in the finite element method, Math. Comp. 31 (1977), 94-111.

2. C. N. Dawson and Q. Du, A finite element domain decomposition method for parabolic equations, Rice Technical Report TR90-21, Dept. of Mathematical Sciences, Rice University, Houston, Texas.

3. C. N. Dawson, Q. Du, and T. F. Dupont, A finite difference domain decomposition algorithm for numerical solution of the heat equation, Math. Comp. 57 (1991), 63-71.

4. C. N. Dawson and T. F. Dupont, Explicit/implicit conservative domain decomposition procedures for parabolic problems in one space dimension (in preparation).

5. T. F. Dupont and P. Keenan, An a priori estimate for variable-time-step second-order backward difference methods (in preparation).

6. M. F. Wheeler, A priori $L_{2}$ error estimates for Galerkin approximations to parabolic partial differential equations, SIAM J. Numer. Anal. 10 (1973), 723-759.

Department of Mathematical Sciences, Rice University, Houston, Texas 77251-1892

E-mail address: clint@rice.edu

Department of Computer Science, University of Chicago, Chicago, Illinois 60637

E-mail address: dupont@tartarus.uchicago.edu 